\title{
Dynamics at the Liquid-Vapor Interface of a Supercooled Organic Glass Former
}

\author{
M. Sikorski, ${ }^{1, *}$ C. Gutt, ${ }^{1}$ Y. Chushkin, ${ }^{2}$ M. Lippmann, ${ }^{1}$ and H. Franz ${ }^{1}$ \\ ${ }^{1}$ Deutsches Elektronen Synchrotron (HASYLAB at DESY), Notkestrasse 85, 22607 Hamburg, Germany \\ ${ }^{2}$ European Synchrotron Radiation Facility (ESRF), B.P. 220, 38043 Grenoble Cedex, France \\ (Received 4 September 2009; revised manuscript received 30 March 2010; published 19 November 2010)
}

\begin{abstract}
We investigated the dynamics near the liquid-vapor interface of the supercooled model organic glass former dibutyl phthalate by using surface-sensitive x-ray scattering techniques. Our results reveal significant enhancement of the relaxation rate over a wide length-scales range. The analysis of the dispersion relation of long-wavelength surface fluctuations yields a nonzero value of the share modulus near the free surface. At the molecular level, the dynamics in the near surface region $(10-15 \mathrm{~nm})$ is inhomogeneous. The mobility is decreasing with increasing distance from the free surface. Below the bulk glass transition, two distinct relaxation times were observed differing by 1 order of magnitude. The observed fast relaxation proves the existence of a high mobility liquidlike surface layer of $10 \mathrm{~nm}$ thickness on top of a frozen in bulk system.
\end{abstract}

DOI: 10.1103/PhysRevLett.105.215701

PACS numbers: 64.70.pm

The nature of the supercooled liquid-vapor and glassvapor interfaces is a problem of considerable interest in condensed matter physics. Despite extensive studies involving various experimental approaches, the current picture of the free surface impact on the dynamics and the viscoelastic properties of the liquid and glassy states is very controversial. In a number of reported measurements, an increase of the glass transition temperature $T_{g}$ near the interface has been shown [1], while others postulated an opposite effect [2-6]. Generally, it is believed that the observed features result from the interplay between two mechanisms: (i) the suppression of the molecular mobility due to interaction of the sample with its support and (ii) the enhanced mobility at the free surface. Often the second effect is argued to be accompanied by the existence of a mobile layer on top of bulk glass, although this concept has also been questioned [7]. There is also no agreement concerning the viscoelasticity near the free surface. Even for the same class of systems, polymers and polymeric liquids, reported data are far from being consistent [7-9].

The work presented in this Letter was motivated by the lack of comprehensive studies of the dynamical aspects of the liquid-vapor interface. By probing a wide range of time and length scales, we intended to verify the concepts of the mobile layer formation and finite shear modulus near the surface. With the help of two grazing incidence scattering techniques, we measured directly the surface dynamics of a simple molecular liquid in the supercooled and glassy state. X-ray photon correlation spectroscopy (XPCS) has been used to investigate the slow dynamics of long ranging capillary wave surface motion down to the glass transition temperature $T_{g}$, while quasielastic nuclear forward scattering (QNFS) has been used to study the surface dynamics on molecular length scales around the critical temperature $T_{c}>T_{g}$ introduced by mode-coupling theory [10].
The system under study was the model organic glass former, dibutyl phthalate (DBP) $\mathrm{C}_{16} \mathrm{H}_{22} \mathrm{O}_{4}$ combining simple molecular structure with high glass-forming ability. Properties of supercooled and glassy DBP have been investigated in bulk [11-13] and for confined samples [14,15]. Differential scanning calorimetry (heating rate $10 \mathrm{~K} / \mathrm{min}$ ) and QNFS [16] measurements of the bulk sample yielded a glass transition temperature $T_{g}^{\text {bulk }}=179 \pm 1 \mathrm{~K}$ and a critical temperature $T_{c}^{\text {bulk }}=202 \mathrm{~K}$, respectively.

The experiments reported in this work have been performed with a liquid surface sample cell based on an existing setup [17], modified to provide faster cooling rates along with better temperature stability from 300 down to $95 \mathrm{~K}$. The temperature of the 0.3 -mm-thick DBP films was ramped with an average speed of $0.6 \mathrm{~K} / \mathrm{min}$. After reaching the desired temperature, the sample environment was stabilized for around 30 minutes before the measurement started. Surface sensitivity has been established by setting the incident angle of the x-ray beam to $80 \%$ of the value of the critical angle of total external reflection $\alpha_{c}$ with the help of a vertically tilted Si mirror. The penetration depth of the radiation is limited in this case to a couple of nanometers.

A liquid surface is not flat but corrugated due to the thermally induced fluctuations. The amplitude and the dispersion relation of such waves is determined by the properties of the given liquid, like viscosity $\eta$, surface tension $\gamma$, and elastic modulus $\mu$. XPCS provides a way to study the slow dynamics of the surface waves at the submicrometer length scale. The measured quantity is the normalized intensity autocorrelation function [18]

$$
g_{2}\left(\tau, q_{x}\right)=\frac{\left\langle I\left(t, q_{x}\right) I\left(t+\tau, q_{x}\right)\right\rangle}{\left\langle I\left(t, q_{x}\right)\right\rangle^{2}},
$$

where $I\left(t, q_{x}\right)$ and $I\left(t+\tau, q_{x}\right)$ are the x-ray intensities at time $t$ and time $t+\tau$, respectively, at wave-vector transfer $q_{x}$. 
The XPCS measurements were performed at the beam line ID10A (TROIKA I) of the ESRF. A pinhole with a diameter of $10 \mu \mathrm{m}$ was placed before the sample in order to obtain a collimated and transversely partially coherent beam. Two detectors were used to collect the off-specular intensity. The faster dynamics at higher temperatures was investigated by using a point detector. The $g_{2}\left(\tau, q_{x}\right)$ functions were calculated by a hardware autocorrelator plugged directly to the detector. Below $200 \mathrm{~K}$, the characteristic times of the surface modes $\tau_{0}$ entered the time window suitable for $\mathrm{CCD}$ cameras. For the $\mathrm{CCD}$, the intensity autocorrelation functions were calculated by correlating the intensities at discrete times (frames) at selected values in $q_{x}$ space according to the expression given in Ref. [19]. A slight deviation from a simple exponential relaxation is observed from the analysis of the normalized intensity autocorrelation profiles

$$
g_{2}\left(\tau, q_{x}\right)=1+g_{0} \exp \left[-\left(\tau / \tau_{0}\right)^{0.9}\right] .
$$

The maximum contrast $g_{0}$ of the autocorrelation functions of 0.35 and 0.2 was observed for the point detector and CCD camera measurements, respectively.

The obtained dispersion relations $\tau_{0}\left(q_{x}, T\right)$ of the surface modes are presented in Fig. 1. In the case of Newtonian liquids $(\mu=0)$, so-called capillary waves are present at the interface. Interestingly, the collected data cannot be explained in the framework of the capillarywave model (dotted lines) already at temperature as high as $220 \mathrm{~K}$, i.e., $40 \mathrm{~K}$ above $T_{g}^{\text {bulk }}$. Clearly, beside the viscosity, also the elastic properties of dibutyl phthalate must be taken into account in the analysis of the XPCS findings. The mismatch between the observed values and the capillary-wave model predictions becomes more obvious with decreasing temperature. A pronounced crossover from capillary waves to the viscoelastic type of Rayleigh modes at $q_{x}=1 \times 10^{-4} \mathrm{~nm}^{-1}$ was observed at $195 \mathrm{~K}$. The collected data are well-described in the framework of a Kelvin-Voigt model of a viscoelastic medium (solid lines):

$$
\frac{1}{\tau_{0}}=\frac{\gamma(T)}{2 \eta(T)}\left(q_{x}+\frac{\mu(T)}{\gamma(T)}\right) .
$$

As shown in Fig. 2, the deduced viscosity agrees well with the bulk data measured with inelastic x-ray scattering [12] and ultrasonic measurements [11,13]. Similarly to $\eta(T)$, also for the shear modulus $\mu(T)$ a single VogelFulcher-Tammann equation $\left(T_{0}=149 \mathrm{~K}\right)$ is sufficient to model the XPCS findings.

The viscosity is in good agreement with the bulk values. In an XPCS study Kim et al. reported also a good agreement between bulk and surface viscosity for thin polymer films. However, we also observe pronounced elastic effects. So besides the viscous relaxation the presence of the shear modulus allows us to investigate the $q_{x}$-independent structural relaxation rate $\lambda(T)=\mu(T) / \eta(T)$ (Fig. 2), which was not accessible in the experiment of Kim et al. The relaxation rate for the dibutyl phthalate surface consists of
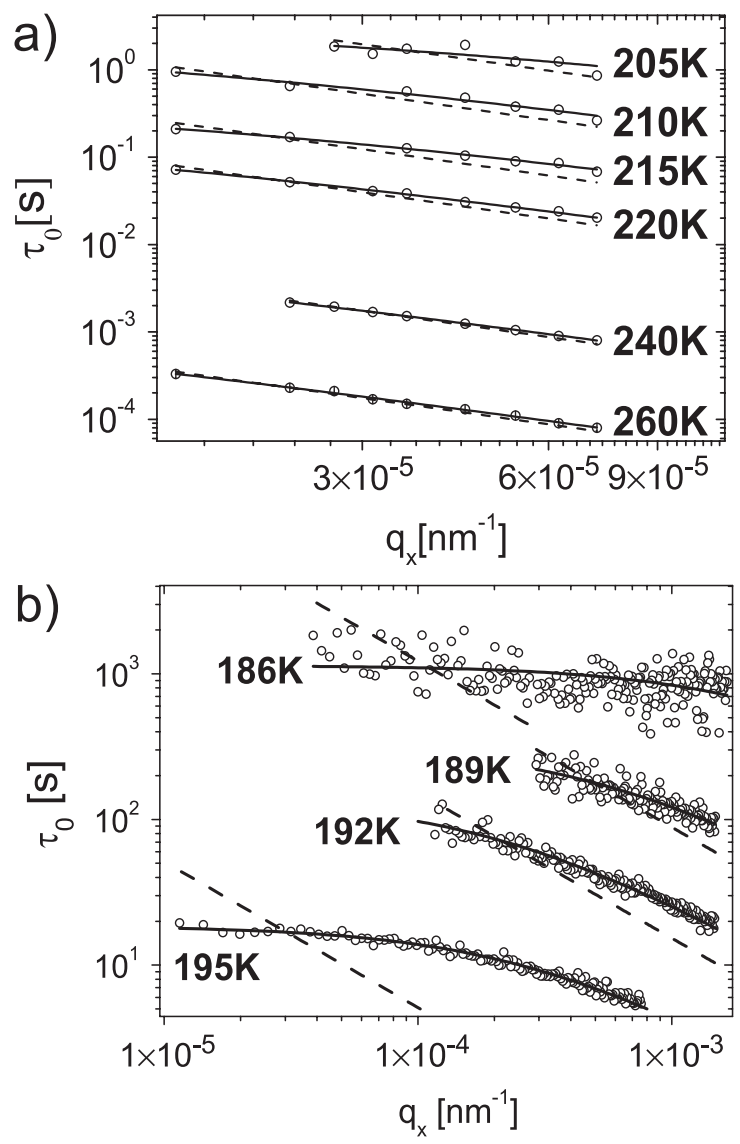

FIG. 1. Dispersion curves $\tau_{0}\left(q_{x}, T\right)$ of capillary waves collected at the free surface of dibutyl phthalate by using (a) the point detector and (b) the CCD camera. The solid line corresponds to fits according to the Kelvin-Voigt model of a viscoelastic medium. The dashed line represent fits with the shear modulus set to zero $(\mu=0)$.

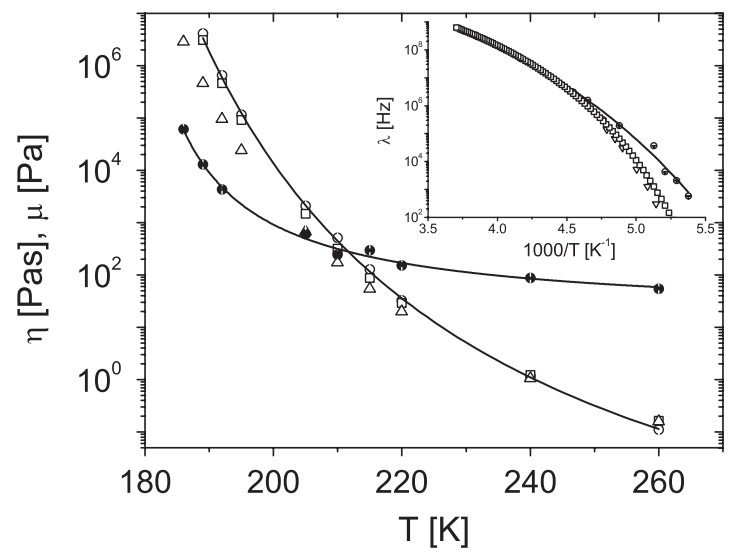

FIG. 2. The viscosity $\eta(T)(\bigcirc)$ and the shear modulus $\mu(T)$ (O) near the free surface compared to the inelastic x-ray scattering [12] $(\triangle)$ and the ultrasonic [11] data $(\square)$ of a bulk sample. Inset: The scaled relaxation rate $\lambda(T)$ calculated from XPCS data (O) compared to Ref. [11] and the dielectric relaxation $(\nabla)$. The solid lines represent the Vogel-Fulcher-Tammann fits. 
a single branch, identified as $\alpha$ relaxation. To compare the temperature dependence of the surface $\lambda$ with the bulk measurements, all plots were scaled to coincide at $260 \mathrm{~K}$. At highest temperatures $(205 \mathrm{~K}<T<260 \mathrm{~K})$, all the data sets coincide. Upon approaching $T_{g}^{\text {bulk }}$ the relaxation rate $\lambda$ near the interface decouples from the bulk data and is 1 order of magnitude greater than deep inside the liquid.

The aim of the QNFS experiment was to study the surface relaxation processes at the nanometer length scale

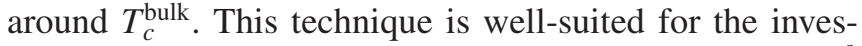
tigation of molecular motion in the time window from $10^{-8}$ to $10^{-6} \mathrm{~s}$ corresponding to the transition from the fast $\beta$ to the $\alpha$ relaxation. In a QNFS experiment the coherent decay of the excited state of an ensemble of ${ }^{57} \mathrm{Fe}$ tracers is monitored as a function of time after an exciting synchrotron pulse. The measurements were performed at beam line ID18 of the ESRF, operated in 16-bunch filling mode. The isotope was introduced by doping dibutyl phthalate with ${ }^{57} \mathrm{Fe}$-enriched ferrocene $\mathrm{Fe}\left(\mathrm{C}_{5} \mathrm{H}_{5}\right)_{2}$ with molecular ratio 1:24. The sample was exposed to highly monochromatic $(\Delta E \approx 1.3 \mathrm{meV}) 14.41 \mathrm{keV}$ radiation at an angle of incidence of $1.12 \mathrm{mrad}$ (penetration depth $15 \mathrm{~nm}$ ). Photons reemitted in the forward direction within the time window from 20 to $160 \mathrm{~ns}$ after the exciting prompt pulse were collected with a stack of three avalanche photodiodes. The time spectra were measured for sample temperatures from 149 up to $194 \mathrm{~K}$. Data were evaluated within the distorted wave Born approximation in the kinematic limit [14] assuming the intermediate scattering function to have the stretched exponential form $F_{s}(q, t)=$ $\exp \left[-\left(t / \tau_{0}\right)^{\beta}\right]$, where $\tau_{0}$ denotes the relaxation time (note that this value is measured at a much higher $q$ value than the $\tau_{0}$ measured with XPCS). Since the accessible time window was too narrow to determine the value of the stretching exponent, the value reported for bulk and confined dibutyl phthalate $\beta=0.5[14,16]$ was used. Examples of collected spectra are presented in Fig. 3. Similar to the bulk and confined liquids, the QNFS data collected above $T_{g}^{\text {bulk }}$ were modeled with a single value of the relaxation time $\tau_{0}$ (inset in Fig. 3). Surprisingly, the values of $\tau_{0}$ decrease with decreasing temperature (Fig. 4). However, below $T_{g}^{\text {bulk }}$ a satisfactory fit can be obtained only by assuming a coherent superposition of two QNFS signals characterized by two distinct relaxation times $\tau_{01}(T)$ and $\tau_{02}(T)$ (Fig. 3). The first one remains constant within the experimental error, while the second one increases upon further cooling (Fig. 4). When compared with data reported for bulk samples [14,20], $\tau_{01}$ matches the value observed for glassy DBP, while the value of $\tau_{02}$ corresponds to the supercooled state.

In order to explain such a complex and counterintuitive temperature dependence of the relaxation time, we propose a concept describing the molecular mobility as a function of distance from the interface [2,21]. According to this picture the relaxation time of the molecules depends not only on the temperature $T$ but also on the distance from the surface.

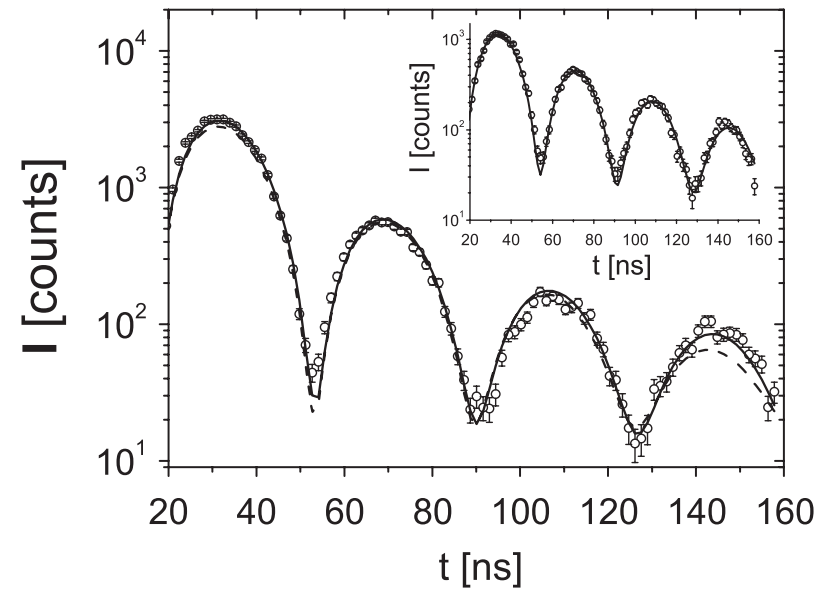

FIG. 3. QNFS pattern collected from the dibutyl phthalateferrocene surface at $170 \mathrm{~K}$. The dashed line is fits with a single relaxation time $\tau_{0}$. The coherent sum of two signals is marked with the solid curve. Inset: Data set collected at $190 \mathrm{~K}$. The solid line represents fit with a single relaxation time.

This model was implemented by using a multilayer model of the liquid-vapor interface. The probed volume has been divided into $15(i=1, \ldots, 15)$ equally thick slices, parallel to the interface and characterized by individual values $\tau_{01 i}(T)$ and the critical temperatures $T_{c}^{i}$. The thickness of the slices was chosen to be equal to the averaged dimensions of DBP molecule. The QNFS signal measured for such a multilayer structure is the coherent sum of the individual resonant signals emitted by each slice:

$$
\tau_{01}(T)=\sum_{i=1}^{15} \omega_{i}(T) \tau_{01 i}(T)
$$

The weight $\omega_{i}(T)$ is proportional to the amplitude of the signals emitted by layer $i$, which is determined by the Lamb-Mössbauer factor $f_{\mathrm{LM}}^{i}(T)$, the intensity of the prompt pulse, and the attenuation of the reemitted photons due to the self-absorption effect. Mode-coupling theory predicts the correct temperature dependence of the LambMössbauer factor $\left[f_{\mathrm{LM}}(T) \propto \sqrt{\left(T_{c}-T\right) / T_{c}}\right]$ for bulk dibutyl phthalate [16]. According to our model, the same relation holds also near the free surface, but the enhancement of the molecular mobility in the vicinity of the interface leads to a decrease in $f_{\mathrm{LM}}^{i}(T)$ due to the shift in $T_{c}^{i}$ to lower values. Above $T_{c}^{i}$, the given layer does not contribute to the observed data. A very steep increase in $f_{\mathrm{LM}}^{i}(T)$ at $T=T_{c}^{i}$ is followed by relatively slow enhancement upon further cooling. As the temperature of the sample is lowered, more and more slices contribute to the signal. Each newly "activated" slice is characterized by a shorter relaxation time than the previous one. As a consequence, the weighted sum, measured during the experiment, becomes smaller. To verify the correctness of the proposed model we calculated $\tau_{01}(T)$ for number of different $T_{c}(i)$ and $\tau_{01 i}(T)$ profiles. Among the relations reproducing the collected data, an exponential increase of the characteristic time scale $\tau_{01 i}=\exp (0.1 i) \exp \left(-10^{3} T\right)$ and linear increase of 


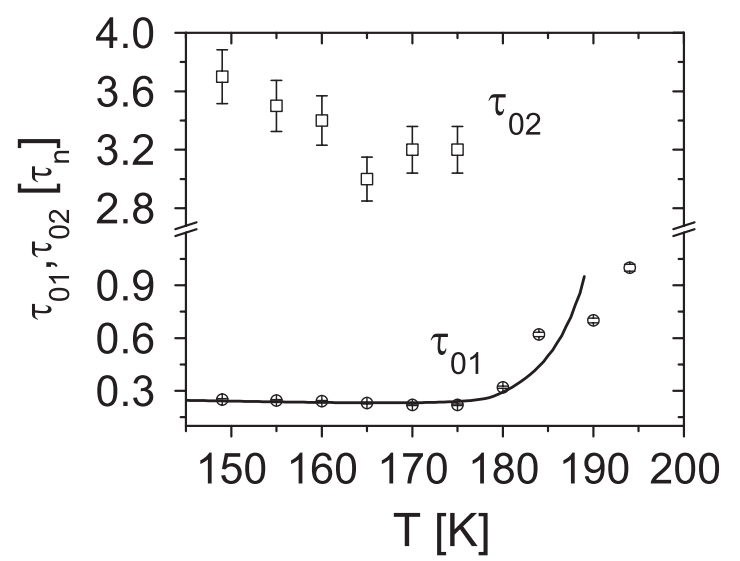

FIG. 4. The relaxation times $\tau_{01} \tau_{02}(T)$ of DBP-ferrocene as a function of temperature $T$. Below $180 \mathrm{~K}$, the data split into the glasslike branch $(\square)$ and the liquidlike branch $(\bigcirc)$. Solid linethe multilayer model prediction of $\tau_{0}$ (for details, see text; $\tau_{n}=$ $141 \mathrm{~ns})$.

the critical temperature $T_{c}^{i}=190 \mathrm{~K}+i \times 0.35 \mathrm{~K}$ are mathematically simplest. The second relation has been chosen such that $\tau_{01 i}(T)$ approaches the bulk value $T_{c}^{\text {bulk }}$ with increasing depth $i$.

Below $T_{g}^{\text {bulk }}$, part of the probed volume freezes while the major fraction remains in the supercooled state. Since vitrification is accompanied by a dramatic slowing-down of the sample dynamics, the distribution of the time scales within the probed volume cannot be approximated by a single time $\tau_{0}$ but is rather approximated by two distinct values $\tau_{01}$ and $\tau_{02}$ (Fig. 3). The constant value of $\tau_{01}$ reflects a very subtle impact of the temperature changes on the observed dynamics in the glassy dibutyl phthalate. On the contrary, the mobility of the supercooled liquid is strongly affected by cooling, and so is the relaxation time $\tau_{02}$. The thickness of around $h=10 \mathrm{~nm}$ at $180 \mathrm{~K}$ and $h=9 \mathrm{~nm}$ at $149 \mathrm{~K}$ of the liquidlike layer has been estimated from the relative amplitudes of two signals. As shown in Fig. 4, the proposed multilayer model provides a good description of the QNFS data and explains all observed features.

The outcome from the experiments described above points towards a very significant impact of the liquid-vapor interface on the properties of the dibutyl phthalate. Although both measurements are sensitive to very different time and length scales, they yield a picture of the free surface enhancing the molecular mobility as compared to the bulk. Similarly to studies focused on polymers and complex liquids, our results also show no evidence of the free surface altering the viscosity of the supercooled liquid and strongly support the concept of elasticity induced by the free surface [8,9]. Although the nonzero $\mu$ value has been measured for several systems, the detailed picture of viscoelasticity at the liquid-vapor interface is not consistent and requires further, more detailed insight. The presented QNFS data advocate a reduced glass transition temperature at the liquid-vapor interface. The collected data provide evidence of the dynamical heterogeneity near the surface. At a given temperature, the molecular mobility depends on the distance from the interface, similar to the effect of surface melting observed on crystalline surfaces [22]. Partial freezing of the probed volume leads to significant diversity of the observed time scales, which can be approximated by the presence of a mobile layer at the interface.

The presented experiments would not have been performed without the tremendous work done by Frank-Uwe Dill, who designed, commissioned, and took care of the surface scattering apparatus. We thank the BW1 (HASYLAB), ID10, and ID18 (ESRF) beam line teams for their great help and assistance during our experiments. R. Roehlsberger and K. Schlage are acknowledged for constructive discussions.

*Present address: Advanced Photon Source, Argonne National Laboratory, Argonne, IL 60439, USA.

[1] O. K. C. Tsui, in Polymer Thin Films, edited by O. K. C. Tsui and T.P. Russell, Series in Soft Condensed Matter (World Scientific, Singapore, 2008), p. 267.

[2] J. L. Keddie, R. A. L. Jones, and R. A. Cory, Europhys. Lett. 27, 59 (1994).

[3] T. Kajiyama, K. Tanaka, and A. Takahara, Macromolecules 28, 3482 (1995).

[4] Z. Yang et al., Science 328, 1676 (2010).

[5] G. B. DeMaggio et al., Phys. Rev. Lett. 78, 1524 (1997).

[6] J. A. Forrest et al., Phys. Rev. Lett. 77, 2002 (1996).

[7] H. Kim et al., Phys. Rev. Lett. 90, 068302 (2003).

[8] Z. Jiang et al., Phys. Rev. Lett. 98, 227801 (2007).

[9] Y. Chushkin, C. Caronna, and A. Madsen, Europhys. Lett. 83, 36001 (2008).

[10] T. R. Kirkpatrick, Phys. Rev. A 31, 939 (1985).

[11] L. R. Cook et al., J. Chem. Phys. 100, 5178 (1994).

[12] A. Mermet et al., Phys. Rev. E 66, 031510 (2002).

[13] A. J. Barlow, J. Lamb, and A. J. Matheson, Proc. R. Soc. A 292, 322 (1966).

[14] T. Asthalter et al., Eur. Phys. J. B 12, S9 (2003).

[15] G. Wellenreuther et al., Hyperfine Interact. 165, 141 (2006).

[16] I. Sergueev et al., Phys. Rev. B 66, 184210 (2002).

[17] T. Seydel et al., Rev. Sci. Instrum. 74, 4033 (2003).

[18] B. J. Berne and R. Pecora, Dynamic Light Scattering: With Applications to Chemistry, Biology, and Physics (Dover, New York, 2000).

[19] D. Lumma et al., Rev. Sci. Instrum. 71, 3274 (2000).

[20] T. Asthalter et al., Eur. Phys. J. B 22, 301 (2001).

[21] E. A. Jagla and E. Tosatti, Europhys. Lett. 51, 648 (2000).

[22] H. Dosch, A. Lied, and J. H. Bilgram, Surf. Sci. 327, 145 (1995). 